\title{
SIRT1 confers protection against ischemia/reperfusion injury in cardiomyocytes via regulation of uncoupling protein 2 expression
}

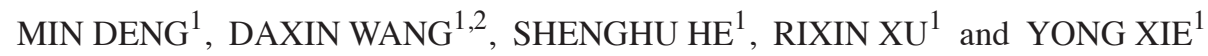 \\ ${ }^{1}$ Department of Cardiology, Subei People's Hospital, Medical College of Yangzhou University, Yangzhou, Jiangsu 225001; \\ ${ }^{2}$ Department of Cardiology, Second Xiangya Hospital, Central South University, Changsha, Hunan 410011, P.R. China
}

Received October 24, 2015; Accepted November 22, 2016

DOI: $10.3892 / \mathrm{mmr} .2017 .7452$

\begin{abstract}
The development of a novel targeted therapy for acute myocardial infarction (AMI) remains a major hurdle in the treatment of cardiovascular disease. Previous studies indicate that mitochondrial uncoupling protein 2 (UCP-2) is involved in the progression of AMI. The present study uses lentivirus knockdown of Sirtuin 1 (SIRT1) in H9c2 cells under hypoxia conditions, and revealed that levels of SIRT1 are accompanied by the expression of UCP-2. Therefore, it was hypothesized that SIRT1 might be important in the development of myocardial infarction. The present study demonstrated that: i) exogenous expression of SIRT1 in vitro induced resistance to hypoxic injury in $\mathrm{H} 9 \mathrm{c} 2$ cells, coinciding with a reduction in expression of UCP-2; ii) knockdown of UCP-2 conferred resistance to hypoxic injury in $\mathrm{H} 9 \mathrm{c} 2$; iii) intraperitoneal injection of resveratrol and the resultant increase in SIRT1 levels may protect against ischemia/reperfusion injury in vivo, concomitant with decreased expression of UCP-2. These findings provide direct evidence that the SIRT1/UCP-2 axis might be important in myocardial infarction, and suggest that this axis may be a novel therapeutic target for the treatment of cardiovascular disease.
\end{abstract}

\section{Introduction}

Cardiovascular diseases are a global cause of mortality, and acute myocardial infarction (AMI) affects $>1.5$ million people in the United States annually (1). The effects of AMI are usually attributable to the detrimental effects of acute myocardial ischemia-reperfusion injury. In the process of myocardial ischemia/reperfusion injury mitochondrial function is disturbed, ROS overexpression and finally causing oxidative stress, energy metabolism disorder, inflammation and ion channel dysfunction, which may lead to DNA oxidation, promoting chain reactions of membrane lipid peroxidation.

Correspondence to: Dr Min Deng, Department of Cardiology, Subei People's Hospital, Medical College of Yangzhou University, 98 Nantong West Road, Yangzhou, Jiangsu 225001, P.R. China

E-mail: dengm2046@sina.com

Key words: SIRT1, uncoupling protein 2, H9c2, hypoxia, resveratrol
Mitochondrial uncoupling proteins (UCPs) are mitochondrial inner membrane proteins that maintain calcium homeostasis and dissipate the proton electrochemical gradient, resulting in the generation of heat, decreased ATP production and reduced generation of mitochondrial reactive oxygen species (ROS) (2). UCP-2 is a member of the UCP family and has functions including regulation of insulin metabolism, modulation of mitochondrial $\mathrm{Ca}^{2+}$ uptake and modulation of apoptosis (3).

Sirtuin 1 (SIRT1) is a nicotinamide adenine dinucleotide-dependent histone deacetylase (4). It has multiple biological functions, including transcription regulation, cell cycle regulation and anti-apoptosis functions (5). SIRT1 represses UCP-2 by binding directly to the UCP-2 promoter (6), and its activation mediates sildenafil-induced delayed cardioprotection against ischemia-reperfusion injury in mice (7).

The aim of the present study was to explore the functional significance of the SIRT1-UCP-2 axis in hypoxia-induced myocardial infarction.

\section{Materials and methods}

Cell culture and in vitro ischemia injury model. Q293T was obtained from the Cell Bank of Type Culture Collection of Chinese Academy of Sciences (Shanghai, China) and used for lentivirus amplification. Rat H9c2 cell lines were obtained from the ATCC (American Type Culture Collection, Manassas, VA, USA), cultured in high glucose Dulbecco's modified Eagle's medium (Gibco; Thermo Fisher Scientific, Inc., Waltham, MA, USA) and supplemented with $10 \%$ (v/v) fetal bovine serum (FBS; Gibco/Invitrogen; Thermo Fisher Scientific, Inc.), $100 \mu \mathrm{g} / \mathrm{ml}$ penicillin, $100 \mu \mathrm{g} / \mathrm{ml}$ streptomycin at $37^{\circ} \mathrm{C}$ in a humidified incubator with $5 \% \mathrm{CO}_{2}$.

When the H9c2 cells were cultured to $70-80 \%$ confluence, the medium was changed to Krebs-Ringer Bicarbonate buffer $\left(115 \mathrm{mM} \mathrm{NaCl}, 4.7 \mathrm{mM} \mathrm{KCl}, 2.5 \mathrm{mM} \mathrm{CaCl}_{2}, 1.2 \mathrm{mM} \mathrm{KH}_{2} \mathrm{PO}_{4}\right.$,

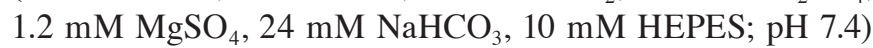
and cultured at $95 \% \mathrm{~N}_{2}$ and $5 \% \mathrm{CO}_{2}$, creating an anoxic environment to stimulate hypoxia (8).

In vitro cell growth assays. When the cells were cultured to $70-80 \%$ confluence, SIRT1 or UCP2 shRNA were transfected into the medium to overexpress SIRT1 or decrease expression of UCP2, using Lipofectamine 2000 (Thermo Fisher Scientific, Inc.). $24 \mathrm{~h}$ following transfection, cells underwent 
$2 \% \mathrm{O}_{2}$ hypoxia treatment for $2,4,6,8,12$ or $24 \mathrm{~h}$. Cell number was then counted under a light microscope following trypan blue staining. All counts were performed on triplicate wells and repeated in three independent experiments at x100 magnification with Image J (version, 1.6.0.24; National Institutes of Health, Bethesda, MD, USA).

In vitro cell survival was measured by 3-(4,5-dimethylthiazol-2-yl)-2,5-diphenyltetrazolium bromide (MTT) assay. Cells were treated with $50 \mu \mathrm{M}$ MTT for $4 \mathrm{~h}$ at $37^{\circ} \mathrm{C}$, and the formazan crystals were dissolved with $200 \mu$ l dimethyl sulfoxide for $10 \mathrm{~min}$ at room temperature. Absorbance was measured at a wavelength of $570 \mathrm{~nm}$.

RNA extraction and reverse transcription-quantitative polymerase chain reaction $(R T-q P C R)$ analysis. Total RNA from heart tissue treated with or without I/R and H9c2 cells were extracted using TRIzol reagent (Invitrogen; Thermo Fisher Scientific, Inc.). RNA concentration was determined by UV spectrophotometer (NanoDrop 2000; Thermo Fisher Scientific, Inc., Wilmington, DE, USA), reverse-transcribed using M-MMLV reverse transcriptase with RNasin Ribonuclease Inhibitors (Promega Corporation, Madison, WI, USA) at $42^{\circ} \mathrm{C}$ for $30 \mathrm{~min}, 95^{\circ} \mathrm{C}$ for $5 \mathrm{~min}$. qPCR amplifications were carried out using the StepOnePlus ${ }^{\text {TM }}$ Real-Time PCR System (Applied Biosystems; Thermo Fisher Scientific, Inc.) and Thunderbird SYBR Master Mix (Toyobo Co., Ltd, Osaka, Japan). Primer sequences were: UCP-2, forward 5'-TGTGGTAAAGGTCCGCTTCC-3' and reverse 5'-CCC GAAGGCAGAAGTGAAGT-3'; Sirt-1, forward 5'-TGAGGT AGGGTCCCCGTTTA-3' and reverse 5'-TTGAGGCCGGTT TGGCTTAT-3'; and GAPDH, forward 5'-GATCCCGCTAAC ATCAAATG-3' and reverse 5'-GAGGGAGTTGTCATATTT CTC-3'. qPCR was performed with the following cycles: $95^{\circ} \mathrm{C}$ for $10 \mathrm{~min}$, followed by $95^{\circ} \mathrm{C}$ for $15 \mathrm{sec}, 60^{\circ} \mathrm{C}$ for $30 \mathrm{sec}$, and $72^{\circ} \mathrm{C}$ for $30 \mathrm{sec}$ for 40 cycles. GAPDH expression was used as an internal control. $2^{-\Delta \Delta \mathrm{Cq}}$ was calculated for every sample. The mRNA expression levels were indicated with $2^{-\Delta \Delta \mathrm{Cq}}$ and normalized to GAPDH (9).

In-vitro UCP2 gene silencing and retroviral infection of SIRT1. Lentivirus containing Rat UCP-2 shRNA (cat. no. V3SR11242238929649; Dharmacon, Inc.; GE Healthcare Life Sciences, Chalfont, UK) was used for UCP-2 gene silencing, and Lentivirus containing a scrambled sequence (shScramble, VSH11660, Thermo Scientific Open Biosystems) was used as the negative control. Lentivirus was produced in transfected 293T packaging cells as described previously (10).

Q293A cells were transfected with retroviral vectors expressing rat SIRT1 (cat. no. 4331182 Thermo Fisher Scientific, Inc.) using Lipofectamine 2000 (Thermo Fisher Scientific, Inc.), and changed to fresh medium on the following day. Following $30 \mathrm{~h}$ further incubation, retroviral particle-containing supernatant was harvested and filtered through a $0.45 \mu \mathrm{m}$ filter (EMD Millipore, Billerica, MA, USA). H9c2 cells were transduced with virus supernatant. Transduced cells were used for RT-qPCR or western blot assays $48 \mathrm{~h}$ later.

Luciferase reporter assay. To determine whether the entire rat UCP-2 3'-untranslated region (UTR) segment was amplified by PCR, using mouse genomic DNA as a template. The PCR products were inserted into the p-MIR-reporter plasmid (Ambion; Thermo Fisher Scientific, Inc.). $2 \times 10^{5}$ cells were seeded in 6-well plates and transfected with $1 \mu \mathrm{g}$ of firefly luciferase reporter plasmid and $0.5 \mu \mathrm{g}$ of Renilla expression vector as a transfection control (Ambion; Thermo Fisher Scientific, Inc.) for luciferase assays. Cells were collected and measured $48 \mathrm{~h}$ following transfection according to the manufacturer's instructions (Dual-Luciferase Assay System; Promega Corporation, Madison, WI, USA).

ATP level assay and medium pH measurement. ATP levels were measured with a Molecular Probes ATP Determination kit (A22066; Invitrogen; Thermo Fisher Scientific, Inc.). $100 \mu 1$ lysis buffer was added into H9c2 cells to prepare the total lysate at room temperature and was centrifuged at $2380 \mathrm{x} g$ at $4^{\circ} \mathrm{C}$ for $15 \mathrm{~min}$, and the supernatant was retrieved for use in the ATP assay according to the manufacturer's instructions. Protein concentration was measured via bicinchoninic acid (BCA) assay. The $\mathrm{pH}$ of the extracellular medium was measured using $\mathrm{pH}$ meter (pB-10, Sartorius AG, Göttingen, Germany).

Animals and in vivo myocardial ischemia/reperfusion protocol. A total of 30 adult male C57BL/6 mice, 8 weeks old and weighing $30.2 \pm 3.5 \mathrm{~g}$ were supplied by the Model Animal Research Center of Nanjing University (Nanjing, China). All animal experiments were conducted under the guidelines on humane use and care of laboratory animals for biomedical research published by the U.S. National Institutes of Health (NIH Publication No. 85-23, revised 1996). All experimental preparations and protocols involving animals were reviewed and approved by the Animal Care and Use Committee of Nanjing University (Nanjing, China).

Resveratrol stock solution was dissolved in DMSO (Sigma-Aldrich; Merck Millipore, Darmstadt, Germany) and then diluted into $5 \mathrm{mg} / \mathrm{ml}$ with saline, and the DMSO was less than $5 \%$. Resveratrol $(5 \mathrm{mg} / \mathrm{ml})$ or saline was injected intraperitoneally (i.p.) $24 \mathrm{~h}$ prior to subjection to $1 \mathrm{~h}$ of myocardial ischemia and $24 \mathrm{~h}$ of reperfusion. Mice were anesthetized with pentobarbital sodium $(60 \mathrm{mg} / \mathrm{kg})$. Ischemia was achieved through ligation of the left anterior descending coronary artery (LAD) using an 8-0 silk suture with a section of PE-10 tubing placed over the LAD, $1 \mathrm{~mm}$ from the tip of the normally positioned left atrium. Following ischemia for $1 \mathrm{~h}$, reperfusion was initiated by releasing the ligature. Sham-operated control mice underwent the same surgical procedures except that the suture placed under the left coronary artery was not tied. A total of 30 animals were included and randomly distributed into the sham, I/R and RES+IR groups, with 10 animals in each group.

Hearts were harvested $24 \mathrm{~h}$ subsequent to this directly for measurement of infarct size and stored in liquid nitrogen immediately, and was processed using TRIzol (Thermo Fisher Scientific, Inc.) for RT-qPCR assays or radioimmunoprecipitation assay buffer (cat. no. 89900; Thermo Fisher Scientific, Inc.) for western blot assays.

Measurement of infarct size. Following reperfusion, myocardial infarct size was evaluated by tetrazolium chloride (TTC) staining. The hearts were immediately removed and sectioned 

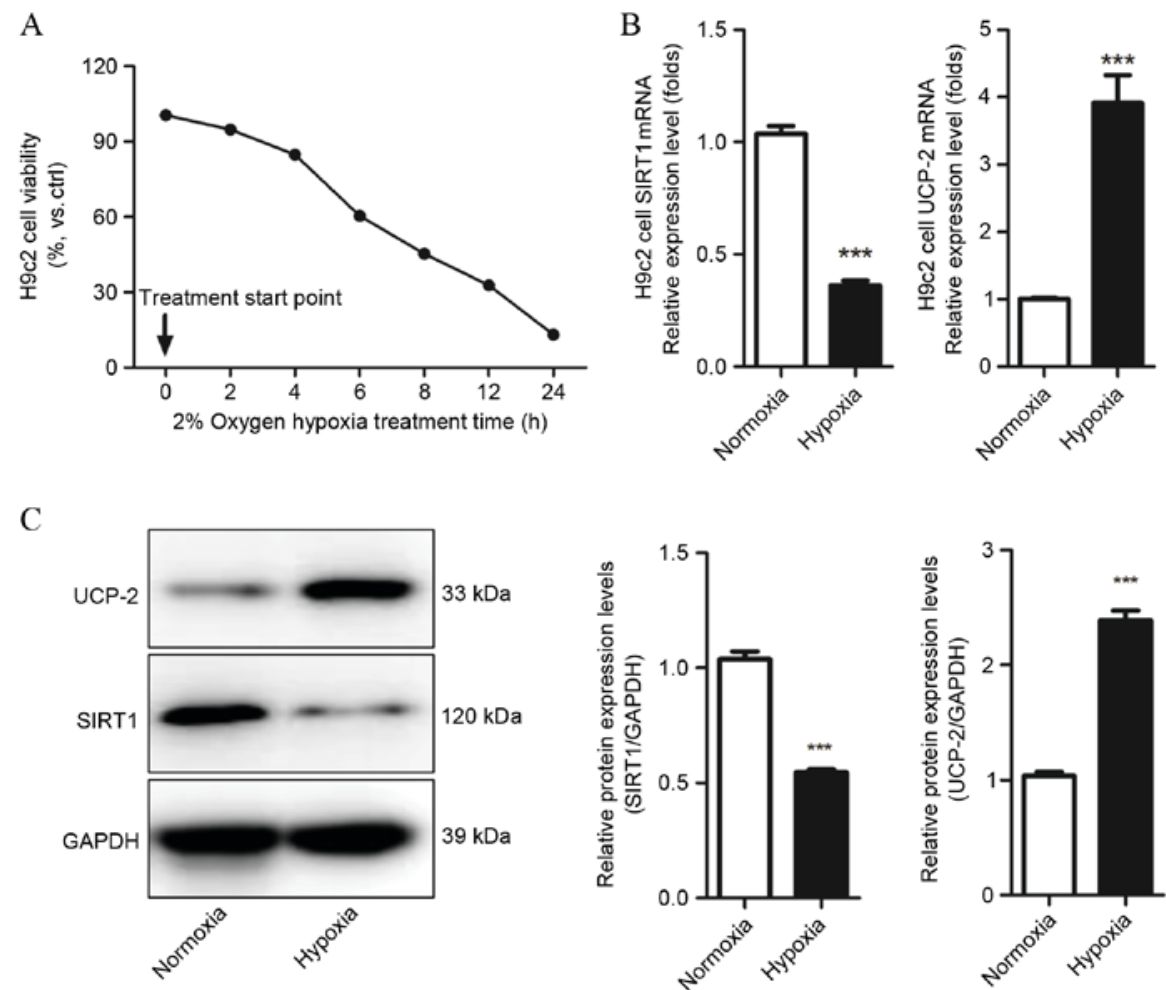

Figure 1. Decreased expression of SIRT1 is concomitant with increased expression of UCP-2 in hypoxia treated cardiovascular cell line H9c2. (A) Viability of rat myocardial $\mathrm{H} 9 \mathrm{c} 2$ cells following $2 \% \mathrm{O}_{2}$ hypoxia treatment. (B) Reverse transcription-quantitative polymerase chain reaction analysis of the relative mRNA expression levels of SIRT1 and UCP2 in H9c2 cells, normalized to GAPDH. (C) Western blot analysis of SIRT1 and UCP-2 protein expression levels in H9c2 cells. GAPDH was used as a protein loading control. Data are presented as the mean + standard error. ${ }^{* * * *} \mathrm{P}<0.001$ vs. normoxia. SIRT1, Sirtuin 1; UCP-2, mitochondrial uncoupling protein 2; GAPDH, glyceraldehyde 3-phosphate dehydrogenase.

into $5 \mathrm{~mm}$ thick short-axis sections from the apex towards the base of the heart, then incubated in $1 \%$ TTC for $20 \mathrm{~min}$ at $37^{\circ} \mathrm{C}$. Following this, the sections were photographed with a Nikon D810 digital camera (Nikon Corporation, Tokyo, Japan). Areas of infarct size were measured digitally using Image Pro Plus software 6.0 (Media Cybernetics, Inc., Rockville, MD, USA).

Western blot analysis. Protein concentrations of the complete lysate were analyzed with the Bradford protein assay. Protein extracts were incubated for $10 \mathrm{~min}$ at $100^{\circ} \mathrm{C}$ in sample buffer $(10 \%$ (v/v) glycerol, $5 \%(\mathrm{w} / \mathrm{v})$ sodium dodecyl sulfate (SDS), $0.25 \%(\mathrm{w} / \mathrm{v})$ bromophenol blue, $5 \%(\mathrm{v} / \mathrm{v}) 2$-mercaptoethanol, and $0.0625 \mathrm{M}$ Tris- $\mathrm{HCl}, \mathrm{pH} 6.8$ ) prior to loading onto the gels. A total of $30 \mu \mathrm{g}$ of homogenate protein was loaded into each lane of a 12\% SDS-PAGE mini-gel (Bio-Rad Laboratories, Inc., Hercules, CA, USA). Electrophoresis was conducted at $80 \mathrm{~V}$ in running buffer $(0.025 \mathrm{M}$ Tris-HCl, $0.2 \mathrm{M}$ glycine, $1 \mathrm{mM}$ EDTA and $3.5 \mathrm{mM}$ SDS). Proteins were subsequently transferred to a polyvinylidene fluoride membrane (GE Healthcare Life Sciences, Chalfont, UK) and blocked at room temperature with 5\% milk in TBS with 5\% Tween-20 (TBST) for $1 \mathrm{~h}$. Western blotting was performed with mouse monoclonal to GAPDH primary antibody (1:2000; ab8245; Abcam, Cambridge, UK), polyclonal mouse anti-UCP2 primary antibody (1:1,000; ab67241; Abcam) or mouse anti-SIRT1 primary antibody (1:1,000; ab104833; Abcam), followed by goat anti-mouse horseradish peroxidase-conjugated secondary antibody (sc-3791; Santa Cruz Biotechnology, Inc., Dallas, TX,
USA) at a dilution of 1:5,000. Following washing, the bands were detected by an enhanced chemiluminescence reagent (EMD Millipore). The band intensities were quantified using the Quantity One 1-D software (version, 4.6.6) in ChemiDoc ${ }^{\mathrm{TM}}$ MP System (Bio-Rad Laboratories, Inc.).

Statistical analysis. All results were expressed as the mean + standard error from at least three independent experiments. Student's $t$-test was used for normally distributed data, and the Mann-Whitney U test was used for abnormally distributed data. $\mathrm{P}<0.05$ and $\mathrm{P}<0.01$ were considered to indicate a statistically significant and a highly significant difference, respectively.

\section{Results}

Decreased expression of SIRT1 is concomitant with increased expression of UCP-2 in hypoxic rat cardiomyocytes (H9c2). Cell viability was detected by MTT assays and cell counting to evaluate whether $\mathrm{H} 9 \mathrm{c} 2$ cells were suffering from hypoxic injury, and determine which hypoxia condition should be used in subsequent experiments. Hypoxia markedly decreased H9c2 cell viability and number as time increased, and following $6 \mathrm{~h}$ hypoxia treatment, cell viability decreased to $61.25 \pm 5.14 \%$ compared with the control group ( $\mathrm{P}=0.021 \mathrm{Fig}$. 1A). Therefore, this condition was used in subsequent experiments. SIRT1 and UCP-2 mRNA and protein levels in H9c2 under normoxic and hypoxic conditions were evaluated by qPCR and western blot assays. Decreased expression of SIRT1 mRNA and protein 
A
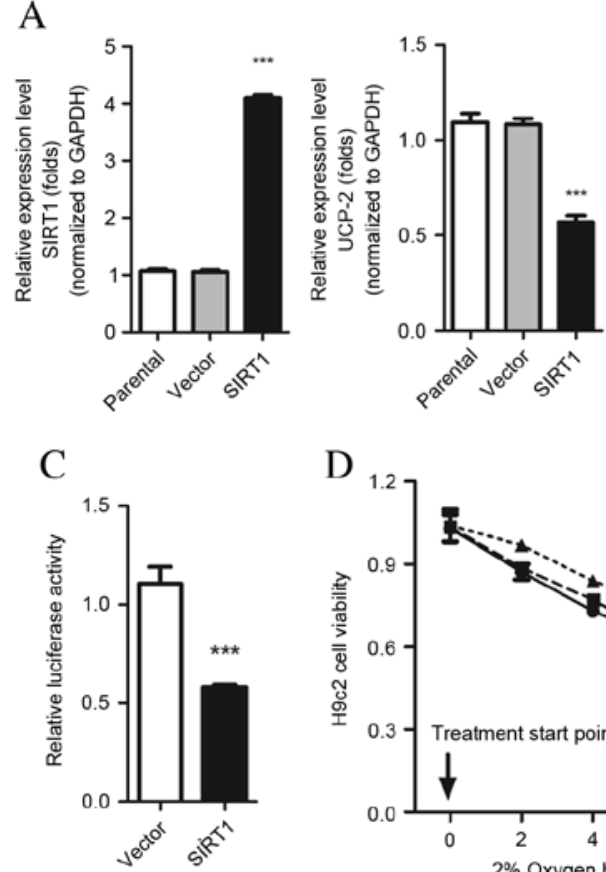

D

\section{B}

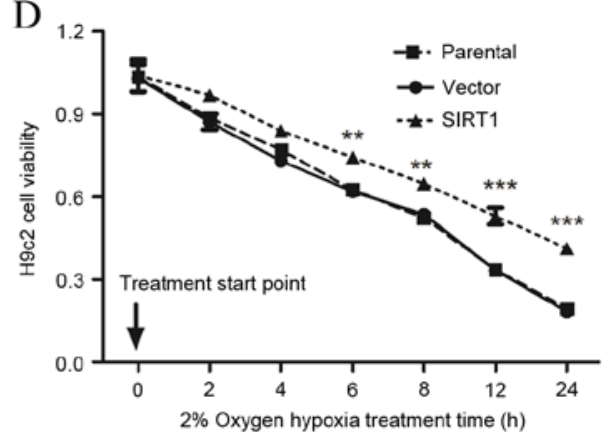

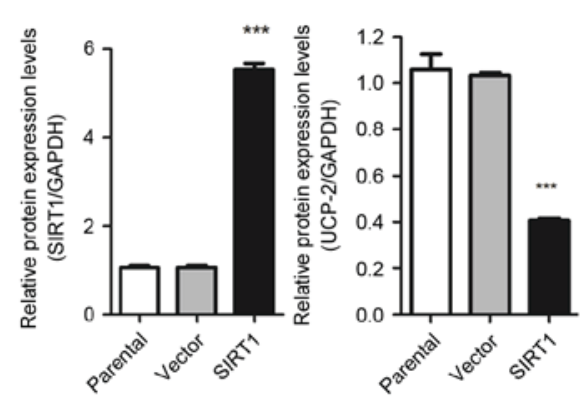

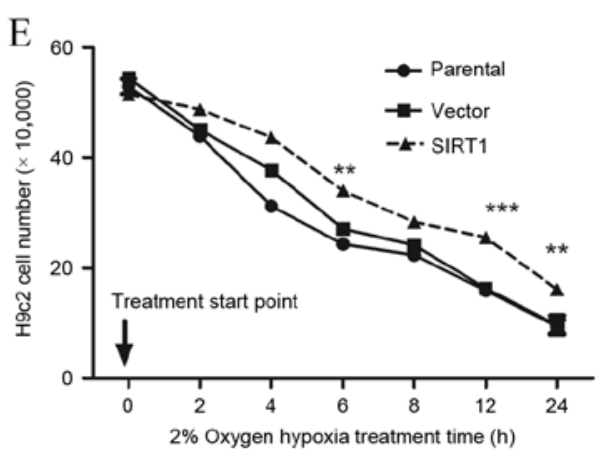

Figure 2. SIRT1 sensitizes the hypoxia response in cardiovascular cell line H9c2 via direct regulation of UCP-2 expression. (A) H9c2 cells were transfected with Lentiviral vector overexpressing SIRT1 and empty vector control. SIRT1 and UCP-2 (A) mRNA and (B) protein expression levels were detected by reverse transcription-quantitative polymerase chain reaction and western blot, respectively, $48 \mathrm{~h}$ following transfection. (C) Firefly luciferase reporters containing UCP-2 3'-UTR were co-transfected into H9c2 cells and dual luciferase assays performed $24 \mathrm{~h}$ following transfection. (D) H9c2 cell viability following transfection with scramble control or SIRT1 Lentivirus and treatment with $2 \% \mathrm{O}_{2}$ hypoxia for different lengths of time, detected by MTT assay. (E) Cell number following hypoxia treatment was determined by trypan blue staining. Data are presented as the mean + standard error. The results displayed were repeated in three independent experiments. ${ }^{* * *} \mathrm{P}<0.001$ vs. normoxia group. SIRT1, Sirtuin 1; UCP-2, mitochondrial uncoupling protein 2.

was observed in hypoxic H9c2 cells compared with normoxic H9c 2 cells $(\mathrm{P}=0.0001$ and $\mathrm{P}=0.0004$, respectively; Fig. $1 \mathrm{~B}$ and $\mathrm{C}$, respectively). UCP-2 mRNA and protein levels were significantly increased in hypoxic $\mathrm{H} 9 \mathrm{c} 2$ cells compared with normoxic $\mathrm{H} 9 \mathrm{c} 2$ cells $(\mathrm{P}=0.0008$ and $\mathrm{P}=0.0007$, respectively; Fig. $1 \mathrm{~B}$ and $\mathrm{C}$, respectively). This result demonstrates that SIRT1 and UCP-2 may be involved in the development of hypoxia-induced injury in cardiomyocytes.

Overexpression of SIRT1 results in increased cell survival following hypoxic injury by directly regulating UCP-2 expression. The effect of SIRT1 in hypoxia-induced cardiomyocyte injury was explored using gain-of-function methods in $\mathrm{H} 9 \mathrm{c} 2$ cells. UCP-2 mRNA and protein expression was significantly downregulated in $\mathrm{H} 9 \mathrm{c} 2$ cells overexpressing SIRT1, compared with scramble control cells $(\mathrm{P}=0.0003$ and $\mathrm{P}=0.0002$; Fig. 2A and $\mathrm{B}$, respectively). Furthermore, the dual luciferase assay revealed that overexpression of SIRT1 in H9c2 cells repressed UCP-2 ( $\mathrm{P}=0.0004$; Fig. 2C). This evidence demonstrates that SIRT1 directly regulates the expression of UCP-2 by binding directly to the UCP-2 promoter. The viability of H9c2 cells overexpressing SIRT1 also increased significantly compared with the parental strain and empty vector control, demonstrated by MTT assay and cell counting $(\mathrm{P}<0.01$; Fig. 2D and $\mathrm{E})$.

Knockdown of UCP-2 in H9c2 cells increases cell survival following hypoxia injury through restoration of ATP biosynthesis. shRNA against UCP-2 was used to block the expression of UCP-2 to study the effects of absence of UCP-2 on cell survival under hypoxic conditions. UCP-2 specific shRNA significantly reduced UCP-2 protein and mRNA expression levels compared with scramble control cells $(\mathrm{P}=0.0008$ and $\mathrm{P}=0.0009$ respectively; Fig. $3 \mathrm{~A}$ and $\mathrm{B}$, respectively). Increased cell viability was observed in $\mathrm{H} 9 \mathrm{c} 2$ cells transfected with UCP-2 shRNA compared with scramble control cells from $4 \mathrm{~h}(\mathrm{P}<0.01$; Fig. 3C) demonstrating that UCP-2 knockdown improves cell resistance to hypoxia.

This enhanced cell survival in response to hypoxia treatment, induced by the decreased expression of UCP2, may have been mediated by the regulation of ATP biosynthesis. Under normoxia, there was no significant difference between the UCP-2 shRNA and scramble shRNA groups (11). However, increased ATP accumulation under hypoxic conditions was revealed in the UCP-2 knockdown group compared with the scramble and parental controls $(\mathrm{P}=0.0006$ and $\mathrm{P}=0.0002$ Fig. 3D). In addition, the $\mathrm{pH}$ of the extracellular medium was increased compared to the scramble and the parental controls $(\mathrm{P}=0.0006$ and $\mathrm{P}=0.0003$; Fig. 3D).

SIRT1 increases resistance to ischemia/reperfusion injury in vivo by decreasing UCP-2 expression. Based on the finding that exogenous expression of SIRT1 in H9c2 cells improved resistance to ischemia injury in vitro, potentially through reducing the expression of UCP-2 and therefore increasing ATP levels, the effect of a putative activator of SIRT1, resveratrol, on ischemia/reperfusion injury was explored in vivo. The results demonstrated that treatment with resveratrol 
A

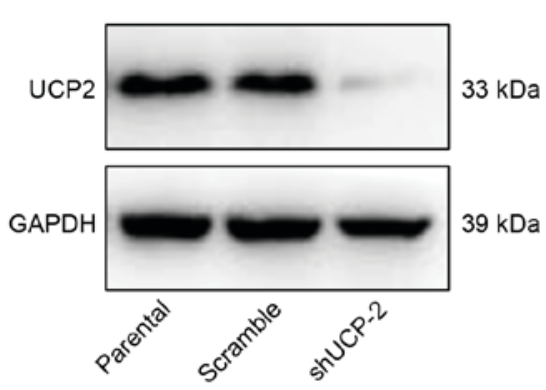

$\mathrm{C}$

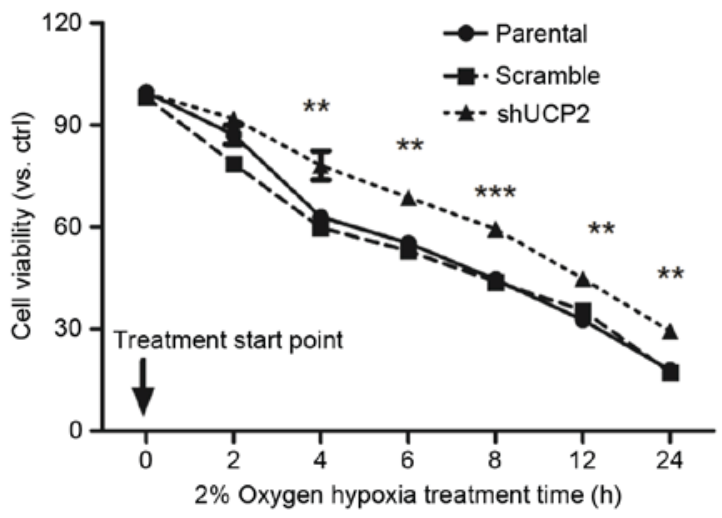

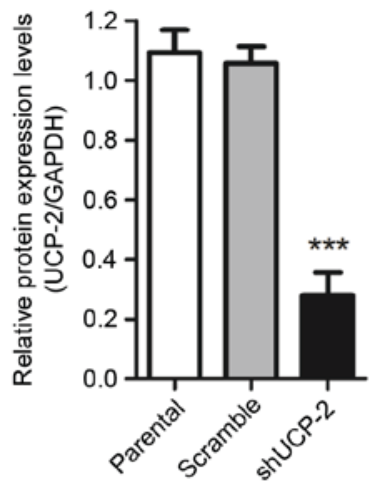

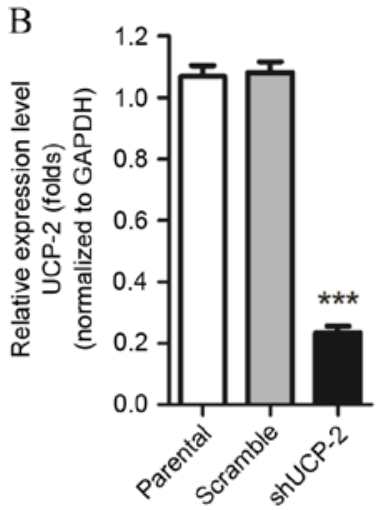

$\mathrm{D}$

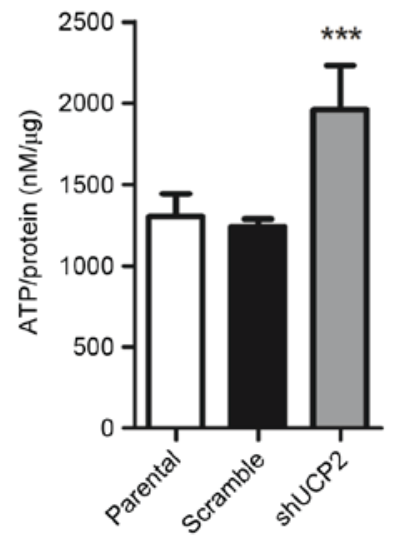

Figure 3. Knockdown of UCP-2 in H9c2 cells results in resistance to hypoxia injury. (A) mRNA and (B) protein expression levels of UCP-2 in H9c2 cells 72 h after transfection with UCP-2 shRNA or scramble shRNA, measured by reverse transcription-quantitative polymerase chain reaction and western blot analysis, respectively, with quantification relative to GAPDH. (C) Cell viability under $2 \% \mathrm{O}_{2}$ hypoxia treatment visualized as a fold change of cell viability normalized to that of normoxia-treated cells. (D) Regulation of extracellular ATP and pH in UCP-2 knockdown H9c2 cells. Data are presented as the mean + standard error. The results displayed were repeated in three independent experiments. ${ }^{* * *} \mathrm{P}<0.001$ vs. scramble control. UCP-2, mitochondrial uncoupling protein 2 ; shRNA, short hairpin RNA.

significantly reduced infarct size compared with the saline control ( $\mathrm{P}=0.003$ Fig. $4 \mathrm{~A})$, suggesting a protective effect against myocardial ischemia/reperfusion injury. Higher mRNA and protein expression levels of SIRT1 in resveratrol-treated myocardial tissue compared with saline controls were validated by RT-qPCR and western blot, respectively, and were concomitant with decreased expression of UCP-2 mRNA and protein $(\mathrm{P}<0.05$; Fig. $4 \mathrm{~B}$ and $\mathrm{C}$, respectively). These findings demonstrate that the SIRT1/UCP2 axis may represent a novel therapeutic target for myocardial ischemia/reperfusion injury treatment.

\section{Discussion}

The present study demonstrated a significant increase in UCP-2 protein expression and reduced SIRT1 protein expression in response to hypoxia treatment in the $\mathrm{H} 9 \mathrm{c} 2$ cell line. Overexpression of SIRT1 in H9c2 cells promoted cell proliferation and confers resistance to hypoxia. Meanwhile, knockdown of UCP-2 by short hairpin RNA (shRNA) in H9c2 cells increased cell proliferation and significantly increased resistance to hypoxia, potentially through increasing ATP levels. SIRT1 was demonstrated to decrease the luciferase activity of the UCP-2 gene promoter and, in vivo, resveratrol-induced increases in SIRT1 levels reduced UCP-2 mRNA and protein levels in a murine ischemia/reperfusion injury model. These findings suggest that SIRT1 may promote hypoxia resistance in cardiomyocytes, mediated via its regulation of UCP-2.

SIRT1 has been demonstrated to protect against cardiovascular diseases, cancer, and neural dysfunction (12-14). Despite its regulatory function in the cell cycle, apoptosis, DNA repair, metabolism and oxidative stress, the involvement of SIRT1 in cardiac cell ischemia/reperfusion injury remains unclear. The present study demonstrated that SIRT1 mRNA and protein levels decreased in response to hypoxia. Furthermore, overexpression of SIRT1 in cardiomyocytes was demonstrated to increase cell survival under hypoxic conditions, indicating that SIRT1 is important in protecting cardiomyocytes during hypoxia.

As a metabolic regulator, UCP-2 protects cardiomyocytes from oxidative stress-induced cell death by reducing the production of ROS in mitochondria (15). Overexpression of UCP-2 in A549 cells has also been demonstrated to promote resistance to hypoxia by inhibiting ROS accumulation and apoptosis (16). However, UCP-2 deficiencies have been demonstrated to increase hepatocyte survival and ATP levels under conditions of ischemia/reperfusion, inhibiting steatosis (2). Prior to the present study no data were available on the function of UCP-2 in cardiac ischemia/reperfusion injury. In the present study, increased expression of UCP-2 
A

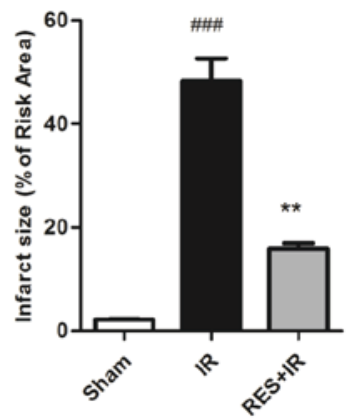

C

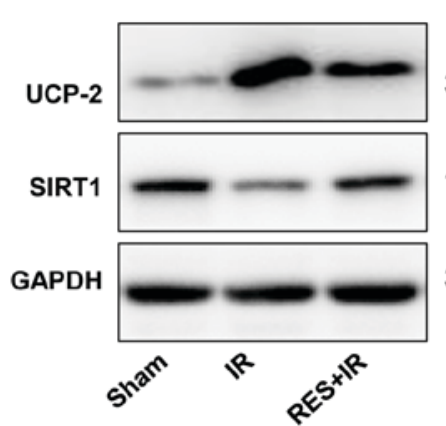

B
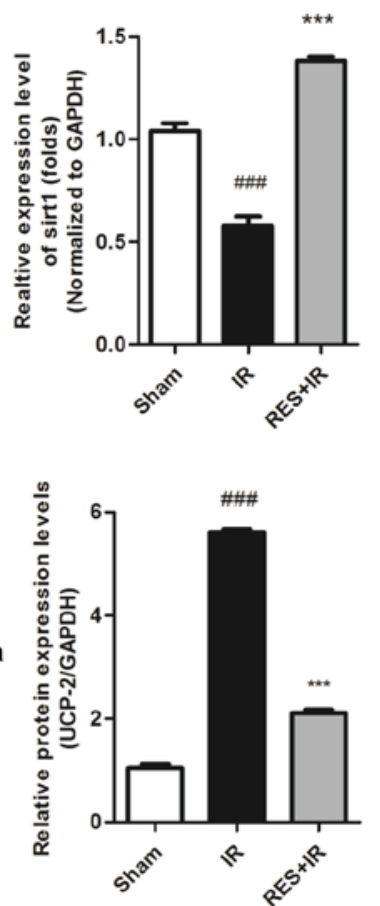
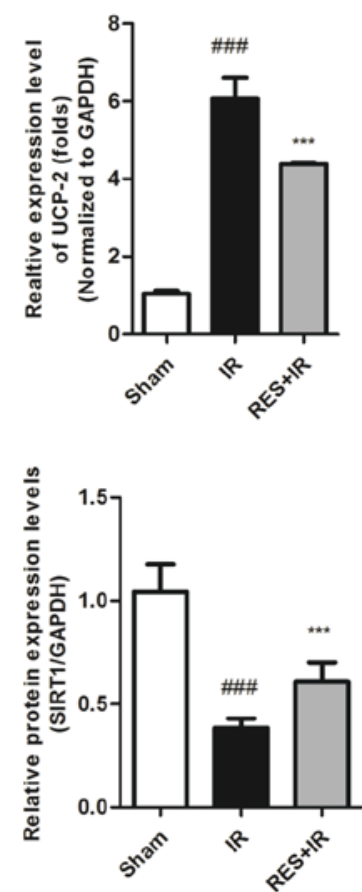

Figure 4. SIRT1 increases resistance to myocardial ischemia/reperfusion injury in vivo by decreasing UCP-2 expression. (A) Myocardial infarct size in C57BL/6 mice treated with $5 \mathrm{mg} / \mathrm{ml}$ intraperitoneal resveratrol $24 \mathrm{~h}$ prior to ischemia/reperfusion injury. (B) SIRT1 and UCP-2 mRNA expression levels were detected by reverse transcription quantitative polymerase chain reaction analysis, with quantification relative to GAPDH. (C) SIRT1 and UCP-2 protein expression levels in the myocardium were determined by western blot analysis, with quantification relative to GAPDH. Data are presented as the mean + standard error. The results displayed were repeated in three independent experiments. ${ }^{\# \#} \mathrm{P}<0.001$ vs. sham group. ${ }^{* * *} \mathrm{P}<0.001$ vs. IR group. SIRT1, Sirtuin 1 ; UCP-2, mitochondrial uncoupling protein 2.

was observed in cardiomyocytes under hypoxic conditions in vitro. This was consistent with previous findings stating that exposure to oxidative stress upregulates and activate UCPs, potentially as a defensive mechanism against oxidative injury $(17,18)$.

However, the present study demonstrated that exogenous expression of SIRT1 resulted in decreased expression of $\mathrm{UCP}-2$, and conferred protection to $\mathrm{H} 9 \mathrm{c} 2$ cells under hypoxic conditions in vitro. Furthermore, the function of UCP-2 was further revealed by the finding that knockdown of UCP-2 increased cell viability, augmented ATP concentration and ameliorated acidosis in $\mathrm{H} 9 \mathrm{c} 2$ cells under hypoxic conditions in vitro. These findings were consistent with a previous study demonstrating the function of the SIRT1/UCP-2 axis in protecting against cerebral ischemia in rats (19). This is direct evidence that the SIRT1/UCP-2 pathway may be a critical component of the development of ischemia/reperfusion injury in cardiomyocytes. To further investigate the effect of SIRT1 on the expression of UCP-2 in H9c2 cells, dual luciferase assay results revealed that overexpression of SIRT1 reduced the transactivation of UCP-2 gene promoters, suggesting that the protective functions of SIRT1 might be mediated by direct regulation of the UCP-2 expression.

Based on the in vitro evidence supporting the involvement of the SIRT1/UCP-2 axis in resistance to hypoxia in $\mathrm{H} 9 \mathrm{c} 2$ cells, the in vivo function of SIRT1 in C57BL/6 mice ischemia/reperfusion injury was investigated. Resveratrol treatment was demonstrated to increase SIRT1 levels, decrease infarct size and led to reduced expression of UCP-2 in cardiomyocytes, which indicated that the SIRT1/UCP-2 axis may be a novel therapeutic target for myocardial infarction treatment. However, UCPs protect against oxidative stress-induced cell death (20), but downregulation of UCP-2 protects against I/R injury, based on the observation of the present results. The extent to which SIRT1/UCP-2 protects against ischemia/reperfusion injury and the paradoxical role of UCP-2 in the heart requires further investigation.

\section{References}

1. Lozano R, Naghavi M, Foreman K, Lim S, Shibuya K, Aboyans V, Abraham J, Adair T, Aggarwal R, Ahn SY, et al: Global and regional mortality from 235 causes of death for 20 age groups in 1990 and 2010: A systematic analysis for the Global Burden of Disease Study 2010. Lancet 380: 2095-2128, 2012.

2. Evans ZP, Palanisamy AP, Sutter AG, Ellett JD, Ramshesh VK, Attaway H, Schmidt MG, Schnellmann RG and Chavin KD: Mitochondrial uncoupling protein-2 deficiency protects steatotic mouse hepatocytes from hypoxia/reoxygenation. Am J Physiol Gastrointest Liver Physiol 302: G336-G342, 2012.

3. Echtay KS: Mitochondrial uncoupling proteins-what is their physiological role? Free Radical Biol Med 43: 1351-1371, 2007.

4. Haigis MC and Guarente LP: Mammalian sirtuins-emerging roles in physiology, aging, and calorie restriction. Genes Dev 20: 2913-2921, 2006

5. Verdin E, Hirschey MD, Finley LW and Haigis MC: Sirtuin regulation of mitochondria: Energy production, apoptosis, and signaling. Trends Biochem Sci 35: 669-675, 2010.

6. Bordone L, Motta MC, Picard F, Robinson A, Jhala US, Apfeld J, McDonagh T, Lemieux M, McBurney M, Szilvasi A, et al: Sirt1 regulates insulin secretion by repressing UCP2 in pancreatic beta cells. PLoS Biol 4: e31, 2006.

7. Shalwala M, Zhu S-G, Das A, Salloum FN, Xi L and Kukreja RC: Sirtuin 1 (SIRT1) activation mediates sildenafil induced delayed cardioprotection against ischemia-reperfusion injury in mice. PLoS One 9: e86977, 2014. 
8. Chiu PY,Luk KF, Leung HY,Ng KM and Ko KM: Schisandrin B stereoisomers protect against hypoxia/reoxygenation-induced apoptosis and inhibit associated changes in $\mathrm{Ca} 2+-$ induced mitochondrial permeability transition and mitochondrial membrane potential in H9c2 cardiomyocytes. Life Sci 82: 1092-1101, 2008

9. Livak KJ and Schmittgen TD: Analysis of relative gene expression data using real-time quantitative PCR and the 2(-Delta Delta C(T)) Method. Methods 25: 402-408, 2001

10. Yuan Y, Yao YF, Hu SN, Gao J and Zhang LL: MiR-133a is functionally involved in doxorubicin-resistance in breast cancer Cells MCF-7 via its regulation of the expression of uncoupling protein 2. PLoS One 10: e0129843, 2015.

11. Bodyak N, Rigor DL, Chen YS, Han Y, Bisping E, Pu WT and Kang PM: Uncoupling protein 2 modulates cell viability in adult rat cardiomyocytes. Am J Physiol Heart Circ Physiol 293: H829-H835, 2007.

12. D'Onofrio N, Vitiello M, Casale R, Servillo L, Giovane A and Balestrieri ML: Sirtuins in vascular diseases: Emerging roles and therapeutic potential. Biochim Biophys Acta 1852: 1311-1322, 2015.

13. Li C, Wang L, Zheng L, Zhan X, Xu B, Jiang J and Wu C: SIRT1 expression is associated with poor prognosis of lung adenocarcinoma. Onco Targets Ther 8: 977-984, 2015.
14. Orozco-Solis R, Ramadori G, Coppari R and Sassone-Corsi P: SIRT1 relays nutritional inputs to the circadian clock through the Sf1 neurons of the ventromedial hypothalamus. Endocrinology 156: 2174-2184, 2015.

15. Teshima Y, Akao M, Jones SP and Marbán E: Uncoupling protein-2 overexpression inhibits mitochondrial death pathway in cardiomyocytes. Circ Res 93: 192-200, 2003.

16. Deng S, Yang Y, Han Y, Li X, Wang X, Li X, Zhang Z and Wang Y: UCP2 inhibits ROS-mediated apoptosis in A549 under hypoxic conditions. PLoS One 7: e30714, 2012.

17. McLeod CJ, Aziz A, Hoyt RF Jr, McCoy JP Jr and Sack MN Uncoupling proteins 2 and 3 function in concert to augment tolerance to cardiac ischemia. J Biol Chem 280: 33470-33476, 2005.

18. Kukat A, Dogan SA, Edgar D, Mourier A, Jacoby C, Maiti P, Mauer J, Becker C, Senft K, Wibom R, et al: Loss of UCP2 attenuates mitochondrial dysfunction without altering ROS production and uncoupling activity. PLoS Genet 10: e1004385, 2014.

19. Della-Morte D, Dave KR, DeFazio RA, Bao YC, Raval AP and Perez-Pinzon MA: Resveratrol pretreatment protects rat brain from cerebral ischemic damage via a sirtuin 1-uncoupling protein 2 pathway. Neuroscience 159: 993-1002, 2009.

20. Teshima Y, Akao M, Jones SP and Marban E: Uncoupling protein-2 overexpression inhibits mitochondrial death pathway in cardiomyocytes. Circ Res 93: 192-200, 2003. 